\section{NOVITÀ IN ENDOCRINOLOGIA}

\section{La medicina che non c'è}

\author{
Silvia Grottoli ${ }^{1}$
}

Accettato: 13 novembre 2021 / Pubblicato online: 21 gennaio 2022

(c) The Author(s) 2022

\section{Commento a:}

\section{La medicina che non c'è.}

\section{O. Davini.}

\section{(2021) Edizioni Dedalo, Bari, p. 96}

"Non si elimina l'incertezza, si negozia con essa", scriveva Edgar Morin nel 2015. La pandemia ha reso evidente la nostra difficoltà di comprendere la scienza e la sua complessità e il caos comunicativo ha evocato una medicina che non c'è. Questo libro è stato scritto con l'intento di imparare a convivere con le inevitabili incertezze e non essere in balia degli istinti o della peggiore politica. Per credere nella vera medicina e non nelle illusioni.

Questa è la breve sintesi di questo testo scritto da un medico per tutti e quindi anche per noi medici.

Partendo dalla pandemia da Sars-CoV-2, Davini mette l'accento su una serie di contraddizioni con cui viene vista la Medicina moderna e che ha generato una sorta di cortocircuito tra la scienza medica e la popolazione. Infatti, per alcuni la Medicina può risolvere ogni nostro problema di salute, mentre per altri la scienza è inutile per fronteggiare le sfide future.

L'autore ha analizzato le molte domande e i molti dubbi che si pongono i comuni cittadini e ha cercato di dare una risposta, almeno parziale. Il libro suggerisce alcuni spunti di riflessione a cui ognuno di noi, medici, non si può sottrarre per meglio comprendere questo malessere che percorre l'Italia negli ultimi tempi.

Bisogna che facciamo i conti con la medicina delle incertezze perché la medicina in sé apre molte porte e risponde a molte domande ma ad ogni domanda risposta, ne nasce un'altra. Perché la sete di conoscenza non finisce mai: una certezza di oggi potrebbe rivelarsi falsa verità in futuro e la scienza è sempre più vicina alla verità che, però, non si è mai certi di raggiungere. Fortunatamente, accorre in nostro

\footnotetext{
S. Grottoli

silvia.grottoli@unito.it

1 Dipartimento di Scienze Mediche, Università degli Studi di Torino, Torino, Italia
}

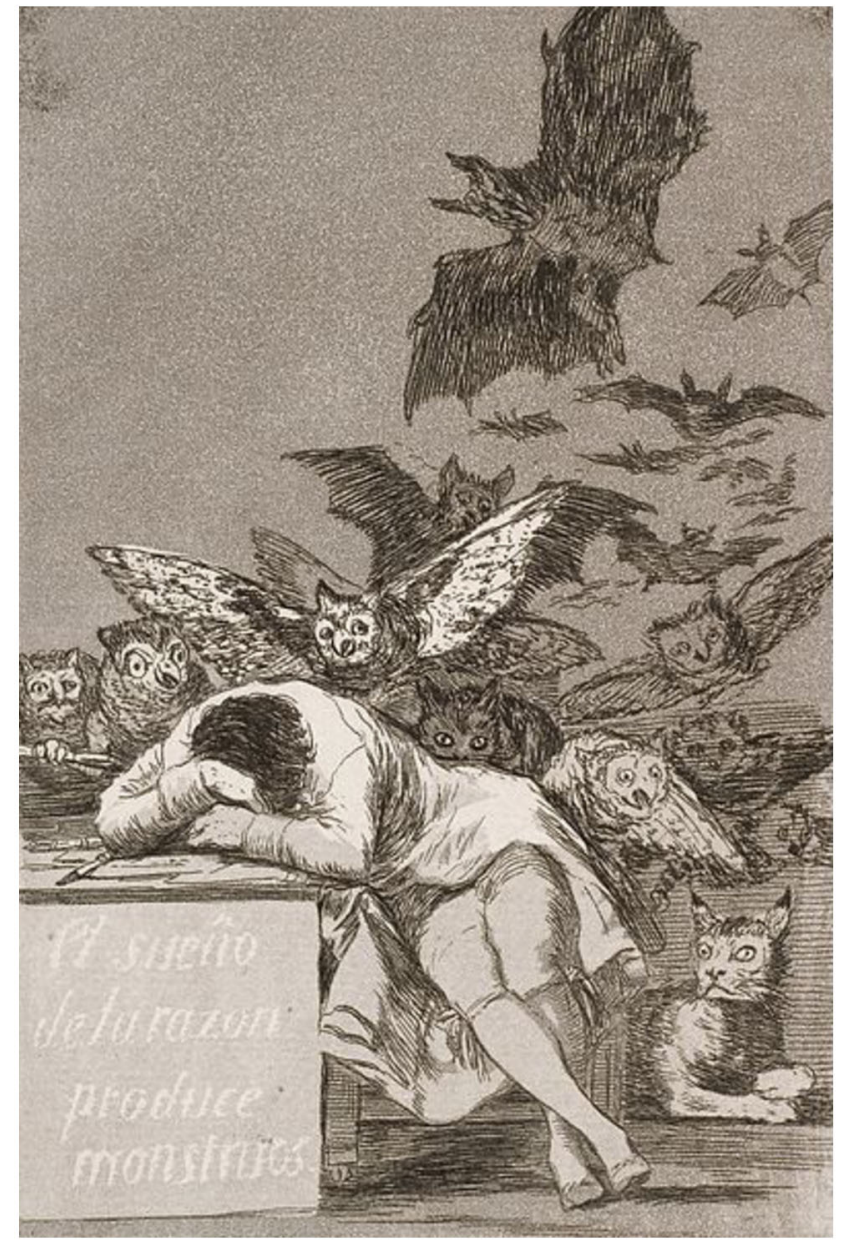

Fig. 1 Francisco Goya (1797) Il sonno della ragione genera mostri, acquaforte, acquatinta e altri mezzi calcografici $(188,98 \times 149,10 \mathrm{~mm})$, The Nelson-Atkins Museum of Art, Kansas City, USA

aiuto il metodo scientifico, che rende la scienza democratica ma rigorosa.

Davini affronta il problema della complessità e della sua differenza rispetto al "complicato/difficile". Il complicato può essere affrontato e risolto con le competenze che, a volte, non sono sufficienti per affrontare un problema complesso. La complessità esprime l'interconnessione dei sistemi, la 
quale permette di far emergere proprietà non possedute dai singoli ma solo dalla loro interazione. A questo argomento si collega il concetto dell' intelligenza cumulativa di gruppo.

Davini accenna e ci suggerisce di approfondire il concetto di One Health, ossia l'interdipendenza tra la salute del pianeta e di chi lo abita.

Infine, il libro affronta la medicina delle illusioni e dell'ignoranza (La medicina che non c'è). L'ignoranza che, in passato, le classi dominanti hanno sempre usato per controllare il popolo e che oggi viene sventolata proprio da coloro che vorrebbero emanciparsi. Ove c'è ignoranza, l'irrazionale prevarrà sempre sul razionale e l'esempio emblematico è quello dei vaccini. Analizzando il concetto di rischio, degli effetti collaterali delle terapie e della sovradiagnosi, l'autore approfondisce il "mito" molto diffuso, ma ovviamente falso, che non si possa più morire. Perché la scienza e la tecnologia di cui disponiamo ci impediranno di soccombere. Da questa credenza, chiaramente smentita negli ultimi due anni, deriva il grande scettiscismo a cui abbiamo e a cui stiamo ancora assistendo nel nostro paese e la conseguente sfiducia nella
Medicina e nella Scienza (Fig. 1).

Il libro è molto più di questo, e vale davvero la pena di leggerlo.

Forse una volta sola non basta ma è un piccolo libro di 90 pagine, con abbondante bibliografia, molto ben scritto e non pesante. Forse leggerlo due volte non ci costerà fatica.

Open Access This article is licensed under a Creative Commons Attribution 4.0 International License, which permits use, sharing, adaptation, distribution and reproduction in any medium or format, as long as you give appropriate credit to the original author(s) and the source, provide a link to the Creative Commons licence, and indicate if changes were made. The images or other third party material in this article are included in the article's Creative Commons licence, unless indicated otherwise in a credit line to the material. If material is not included in the article's Creative Commons licence and your intended use is not permitted by statutory regulation or exceeds the permitted use, you will need to obtain permission directly from the copyright holder. To view a copy of this licence, visit http://creativecommons.org/licenses/by/4.0/.

Nota della casa editrice Springer Nature rimane neutrale in riguardo alle rivendicazioni giurisdizionali nelle mappe pubblicate e nelle affiliazioni istituzionali. 